\title{
GLOBALIZACIÓN, AGROINDUSTRIAS Y AGRICULTURA POR CONTRATO EN MÉXICO*
}

\author{
Flavia Echánove Huacuja ${ }^{1}$ \\ Instituto de Geografía, Universidad Nacional Autónoma de México. \\ Ciudad Universitaria, Circuito Exterior, s/n, México, DF \\ echanovef@yahoo.com
}

\begin{abstract}
Resumen: La agricultura por contrato constituye un mecanismo de abasto de las agroindustrias que se ha expandido aceleradamente en las últimas décadas. En ello han influido los cambios en el consumo, la expansión y fortalecimiento de las agroindustrias y otros distribuidores, y la aplicación de políticas de ajuste estructural. El objetivo de este artículo es el análisis de las características de la agricultura por contrato practicada en torno a la producción de pepinillos de exportación, así como de sus efectos en los productores, mercados de trabajo y de tierras y medio ambiente. Encontramos que los ingresos obtenidos por los productores contratados no los han librado de la necesidad de diversificar sus actividades, y que en general su situación como productores de bienes no-tradicionales es vulnerable y dependiente de los cambiantes escenarios de los mercados globales.
\end{abstract}

Palabras clave: pepinillo, pequeños productores, agricultura por contrato, agroindustrias, México.

\begin{abstract}
Contract farming comprises an agribusiness supply mechanism that has undergone rapid expansion in recent years. Among the factors influencing that expansion are changes in consumption, the growth and strengthening of agribusinesses and other distributors, and the application of structural adjustment policies. The objective of this article is the analysis of the characteristics of the type of contract farming practiced with regard to the production of pickling cucumbers, as well as its effects on producers, labor and land markets, and the environment. We find that the income obtained by contracted producers has not freed them from the necessity of diversifying their activities, and that, in general,
\end{abstract}

\footnotetext{
* Recibido: 27-3-08. Aceptado: 8-7-08.

${ }^{1}$ La presente investigación fue posible gracias al financiamiento de CONACYT (Consejo Nacional de Ciencia y Tecnología), proyecto 45149-S.
} 
their situation as producers of non-traditional commodities is vulnerable and dependent upon global markets.

Keywords: pickles, smallholders, contract farming, agribusiness, Mexico.

\section{Introducción}

La complejidad del sistema de procesamiento y distribución de alimentos durante las últimas décadas se vincula con las nuevas tendencias en el consumo alimentario de los países desarrollados, entre las que destaca la demanda por una mayor variedad, calidad y valor agregado en los alimentos (Perry y Banker, 2000: 50). En términos generales, se asiste a la internacionalización del sistema alimentario mundial y particularmente del sector hortícola, el cual, para responder a dicha demanda, acude cada vez más a la agricultura por contrato. Esta consiste en acuerdos orales o escritos realizados entre los productores directos y diversos agentes (mayoristas, industriales, detallistas, empacadores, organizaciones de productores y empresas públicas), a través de los cuales se regulan diversos aspectos de la producción y comercialización de un producto agrícola. Los acuerdos más comunes son aquellos en que los productores ponen tierra y trabajo, mientras que las empresas aportan material de siembra, insumos químicos y/o asesoría técnica a crédito (Raynolds, 2002: 784; Porter y Phillips-Howard, 1997: 228; Saenz y Ruben, 2004: 140; Echánove y Steffen, 2005). Los precios del producto no siempre se establecen de antemano, pero lo que sí es inherente a la agricultura por contrato es el control directo o indirecto del proceso productivo (Watts, 1994: 26-28; Key y Runsten, 1999: 383; Raynolds, 2000: 441).

Cada vez más productores del Tercer Mundo entran en esquemas contractuales con las agroindustrias, como única posibilidad de acceder a créditos, asesoría técnica, insumos y mercados, ya que la aplicación de políticas de ajuste estructural ha significado la reducción o desaparición de esos apoyos por parte de los gobiernos. En México, la agricultura por contrato prevalece en la producción de azúcar, tabaco, engorda de pollos y cerdos, semillas de granos, cebada, maíz amarillo, ciertas variedades de trigo, y frutas y hortalizas para exportación.

El objetivo de este artículo es analizar las características de la agricultura por contrato practicada en la producción del pepinillo de exportación, así como sus efectos en los productores (nivel de vida), uso del suelo, mercado de trabajo y tierras, y medio ambiente. Dado que dicho régimen laboral representa una institución que vincula procesos locales con globales, llevaremos a cabo un análisis multi-escalar que, de acuerdo a Bebbington (2003), nos permite explicar el escenario local de los productores estudiados, en su relación con decisiones tomadas a otros niveles o escalas geográficas. Elegimos como escenario el estado de Guanajuato, en el cual llevé a cabo durante 2005-2006, entrevistas a profundidad con productores contratados, gerentes de las agroindustrias exportadoras, funcionarios públicos y agentes privados. 


\section{Las hortalizas y el pepinillo en México}

México es un importante productor de hortalizas frescas y el tercer exportador a nivel mundial. Estos bienes se cultivan en el $4 \%$ de la superficie cosechada de ese país, y se exporta solo la cuarta parte de su volumen producido. Sin embargo, para 2007 el valor de la producción de hortalizas representó la quinta parte del generado por el sector agrícola y sus envíos externos contribuyeron con el 70\% de las divisas obtenidas por dicho sector (SAGARPA, 2008), además de ser cultivos relevantes como generadores de empleo, dado que son intensivos en mano de obra.

Durante 1994-2006, el volumen producido de hortalizas aumentó en un 75\% (Gráfica 1), debido mayoritariamente a la expansión del consumo nacional de esos bienes (en un 70\%) y, en menor medida, a la de las exportaciones (30\% restante). Estas se dirigen en cerca del $90 \%$ a Estados Unidos y, como ciertos autores han señalado (Schwentesius y Gómez, 1998: 174-177), se caracterizan por su gran concentración en ciertas regiones, en un grupo de hortalizas y agentes, y en el período de invierno y parte de la primavera. Entre las principales hortalizas producidas en México, el pepino (incluye pepinillo) figura en el noveno lugar en función de su superficie cosechada (Cuadro 1), y el tercero en relación al total de las exportaciones de esos bienes. Su destino es el mercado estadounidense, a donde se enviaron crecientes volúmenes entre 2000 y 2005 (Gráfica 2). Al igual que lo ocurrido con las hortalizas en general, México es el principal abastecedor de pepinos frescos de Estados Unidos (80\% de sus compras externas en 2005).

Gráfico 1. México: superficie cosechada y volumen producido de hortalizas frescas.

\section{Hectáreas}

Miles de tons.

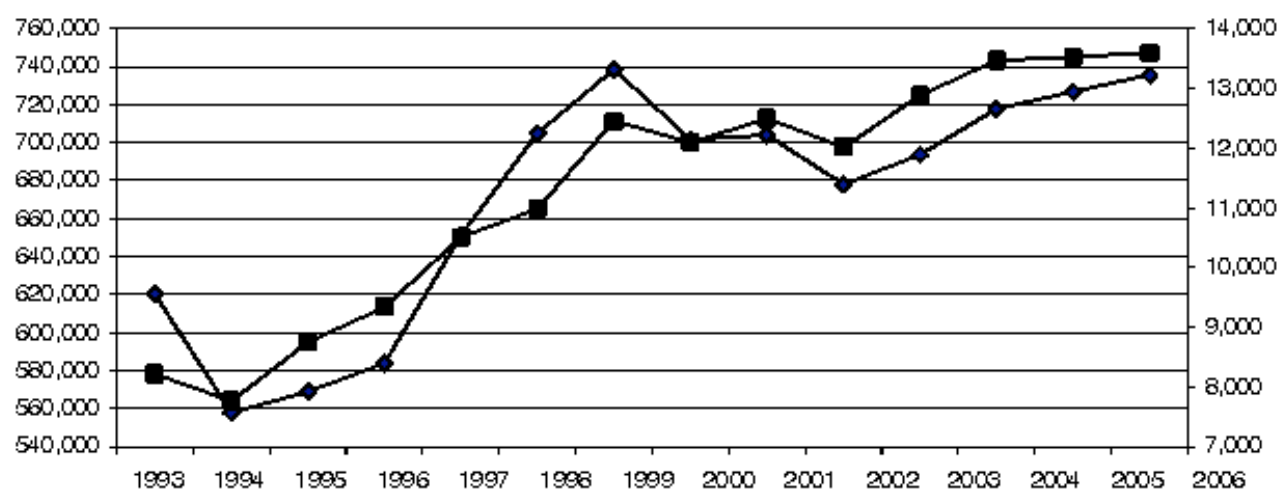

$$
\rightarrow \text {-Superficie } \rightarrow \text { Volumen }
$$

Fuente: Elaborado con cifras de USDA, 2008. 
Cuadro 1. México: principales hortalizas producidas (2006).

\begin{tabular}{lccc}
\hline Producto & $\begin{array}{c}\text { Superficie } \\
\text { cosechada } \\
\text { (bectáreas) }\end{array}$ & $\begin{array}{c}\text { Volumen } \\
\text { de producción } \\
\text { (Miles de toneladas) }\end{array}$ & $\begin{array}{c}\text { Valor } \\
\text { de producción } \\
\text { (Miles de pesos) }\end{array}$ \\
\hline Chile verde & 92,178 & 1,689 & 6,093 \\
Jitomate & 63,061 & 2,093 & 12,314 \\
Tomate verde & 62,593 & 805 & 2,858 \\
Cebolla & 45,673 & 1,238 & 3,585 \\
Sandía & 43,298 & 977 & 1.924 \\
Calabaza y calabacita & 38,669 & 546 & 1.590 \\
Melón & 22,106 & 556 & 1,590 \\
Brócoli & 18,913 & 248 & 881 \\
Pepino y pepinillo & 17,725 & 496 & 1,382 \\
Zanahoria & 14,072 & 359 & 576 \\
Subtotal & 418,288 & 9,007 & 32,974 \\
\hline Total & $\mathbf{7 3 5 , 5 5 1}$ & $\mathbf{1 3 , 5 9 8}$ & $\mathbf{5 1 , 4 6 5}$ \\
\hline
\end{tabular}

Fuente: SAGARPA, 2008.

Gráfico 2. México: exportaciones de pepino y pepinillo fresco a Estados Unidos.

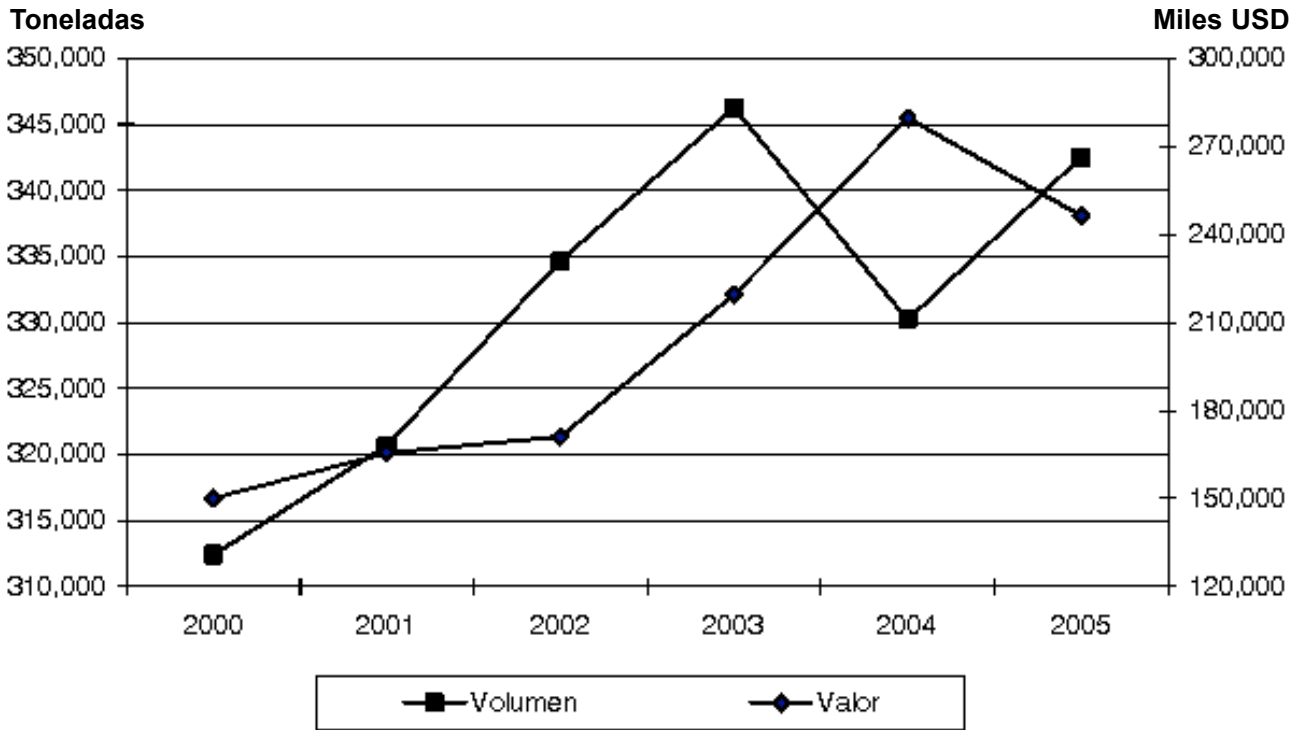

Fuente: Elaborado con cifras de USDA, 2008. 
Los pepinos incluyen dos variedades diferentes de acuerdo a su uso: los que se venden y consumen frescos, y los pepinillos, de menores tamaños y sometidos a un proceso para ser conservados en vinagre. La mayoría de las exportaciones mexicanas de pepinillos son de producto fresco, el cual se procesa en EU, para terminar en los anaqueles de los supermercados y en las hamburguesas preparadas en las cadenas de comida rápida. A pesar de que ese país es un importante productor de pepinillos, tiene escasez, sobre todo de los de tamaño más chico (menores a una pulgada de diámetro), cuyo cultivo es muy intensivo en mano de obra, en especial la cosecha, que es completamente manual. Es en este aspecto en el que países como México e India (principal abastecedor externo de EU de pepinillos procesados), tienen una ventaja comparativa, dados los menores salarios agrícolas pagados, sobre todo en el segundo país, que además cuenta con una mayor oferta de mano de obra.

\section{El pepinillo en Valle de Santiago}

En 2006 se cultivaron en Guanajuato alrededor de 700 hectáreas de pepinillo, la mayoría de las cuales (600 ha) se ubicaron en el municipio de Valle de Santiago, localizado en el sur de esa entidad (Figura 1). El cultivo de esa hortaliza es de ciclo corto (3.5 meses) y solo se lleva a cabo una vez al año porque es muy vulnerable a las lluvias que caracterizan el verano en Valle y a las bajas temperaturas del invierno. Se define como un cultivo "familiar" o "artesanal", dada la gran cantidad de mano de obra que requiere (320 jornadas por hectárea), por lo que lo emprenden pequeños productores en superficies de hasta 5 hectáreas, que se apoyan en el trabajo familiar. Estos son, en su mayoría, miembros de los ejidos de Rincón de Parangueo, San Nicolás, San Isidro, Presa de San Andrés y San Francisco Chihuindo.

Para que no se vea afectada su calidad, los pepinillos requieren de un rápido manejo post-cosecha, teniendo que transportarse directamente desde las parcelas, donde son embolsados en costales de plástico de 30 kilos, a los centros de acopio de las empresas,. En estas instalaciones el producto se pesa, selecciona, embolsa y se embarca en camiones refrigerados con destino a Estados Unidos.

La producción de hortalizas en Valle fue posible en la medida en la que se fueron perforando pozos profundos para extraer agua del subsuelo, y en la que los productores contaron con mercado y financiamiento por parte de las agroindustrias. La primera en establecerse en la zona fue la empresa Campbell's que, aparte de los enlatados, se dedicó al congelamiento de hortalizas y la exportación de pepinillo fresco. Este era enviado a EU, a la empresa Vlasic, que en aquel entonces era la principal elaboradora de "pickles" (pepinillos en salmuera) en ese país y con quien Campbell's tenía una cierta asociación. Para contar con determinada calidad y un abasto seguro, esta empresa hizo contratos con los productores de Valle, a quienes enseñó a cultivar las nuevas hortalizas, desencadenando así un proceso de 
Figura 1. Valle de Santiago, Guanajuato: localización de los ejidos estudiados.

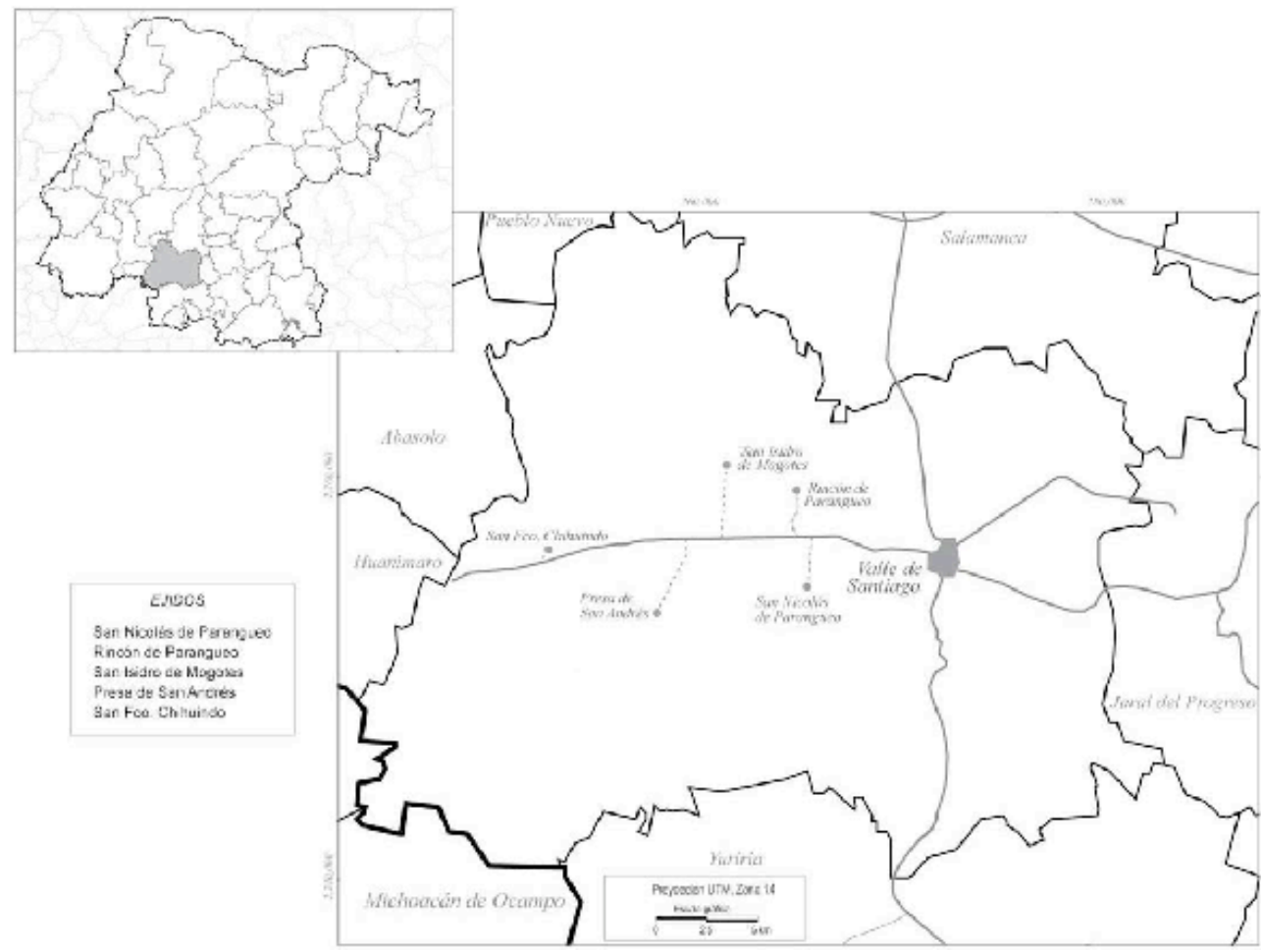

Fuente: elaborado por Laura Luna.

reconversión productiva, bajo el cual numerosas tierras, antes dedicadas a granos, se sembraron con hortalizas. A ello contribuyó el continuo desplome de la rentabilidad de la producción de granos básicos, producto de la supresión de apoyos y subsidios estatales y de la apertura comercial iniciada en México en los años ochenta.

Campbell's enfrentó problemas de mercado y suspendió sus actividades en Valle, pero en 1994 llegó MarBran y Laja Orgánicos $S A$ de $C V$ y, cinco años después, Servicios Agropecuarios El Sol. La historia de MarBran se remonta a 1980, cuando empezó a exportar hortalizas congeladas desde Guanajuato, siendo actualmente la principal empresa de esa rama no solo en México, sino a nivel mundial. En 1992 se asoció con el consorcio alimentario estadounidense $J$. $R$. Simplot y, al poco tiempo, se diversificó hacia la exportación de hortalizas frescas a EU. El pepinillo que exporta MarBran lo adquiere del sur de Guanajuato, de alrededor de 250 hectáreas, de las cuales 190 se ubican en el municipio de Valle y son cultivadas por 100 pequeños 
productores. Al igual que en el resto de las empresas, MarBran envía a sus agrónomos antes de iniciar el período de siembra para contactar a los productores que consideren adecuados. La elección se basa en los recursos que tengan dichos agentes, quienes deben contar con agua para riego y un mínimo de maquinaria y capital, siendo también preferentes aquellos que posean experiencia en el cultivo del pepinillo o de otra hortaliza. Posteriormente, la empresa hace contratos escritos con los productores seleccionados, en los que se especifican las cantidades de producto a entregar, las calidades demandadas y los precios de cada una de éstas. MarBran proporciona a los productores la semilla y los insumos químicos (fertilizantes e insecticidas) a crédito, descontándoles su monto paulatinamente desde la primera entrega de producto que hacen a la empresa. Aunque ésta señala que otorga a los productores asesoría técnica gratuita, algunos de ellos expresaron no haberla recibido nunca, dado que la firma no cuenta con el personal suficiente para ello.

La otra empresa compradora, Servicios Agropecuarios El Sol, propiedad de un estadounidense, se abastece también mediante contratos escritos que especifican cantidades, calidades y precio del producto. Se relaciona con 60 pequeños productores que anualmente cultivan 250 hectáreas. Al igual que MarBran, suministra a los productores la semilla y los insumos químicos a crédito, pero se diferencia en el otorgamiento de una mejor asesoría técnica al productor. Los productores tienen con El Sol otro apoyo que no les brinda MarBran, y que consiste en el préstamo de cierta cantidad de dinero por cada hectárea cultivada para gastos de la siembra y deshierbe y, posteriormente, para cubrir los gastos de mano de obra cuando empieza la cosecha.

Laja Orgánicos, también propiedad de un estadounidense, se relaciona con los productores de manera muy similar a $\mathrm{El} \mathrm{Sol}$, contratando anualmente a alrededor de 70 de ellos, que cultivan en conjunto 160 hectáreas de pepinillo. Su cliente es la empresa estadounidense Bay Valley Foods, principal fabricante y distribuidor de una serie de alimentos procesados para los autoservicios, restaurantes y cadenas de comida rápida de ese país. Estos mismos sitios son también el destino final del pepinillo de las otras dos empresas existentes.

\section{El punto de vista de los productores: ventajas y desventajas de los contratos}

De acuerdo a Singh (2002: 1632), la satisfacción de los productores bajo contrato se mide a través de la rentabilidad de sus cultivos, la eficiencia en los pagos y suministro de insumos, la seguridad del mercado y la participación de los productores en la toma de decisiones. A estos elementos, añadimos aquí la asesoría técnica otorgada por las empresas y las evaluaciones de calidad, las cuales influyen directamente en los niveles de rentabilidad. Respecto a los insumos químicos, los productores de pepinillo entrevistados señalaron que les son cobrados a precios 
superiores a los que prevalecen en las casas comerciales, mientras que las semillas entregadas no siempre tienen buena calidad, lo que redunda en menores rendimientos y calidad del producto final. En cuanto a asesoría técnica, la que proporciona MarBran no beneficia a todos los productores contratados, sin embargo, la otorgada por El Sol y Laja les resulta de gran utilidad.

El aspecto de la calidad suele ser el más conflictivo de la relación entre productores contratados y empresas. En el caso del pepinillo, el primer problema que enfrentan los productores que entregan a MarBran es el hecho de que el mecanismo de recepción del producto es deficiente. Su centro de acopio abre a las 12 am, y desde antes se forman a la orilla de la carretera largas filas de vehículos de los productores que cosecharon su hortaliza unas horas antes, a los que se van agregando los que arriban después. El resultado es la espera bajo el sol durante horas, lo que redunda en un descenso de la calidad del producto, dada la deshidratación a la que se somete. Una vez que éste se recibe, se selecciona por calidad (manual y mecánicamente), y aquí la empresa permite al productor estar presente en este proceso. Cada ocho días se liquida a los productores por las entregas previamente realizadas, descontando en cada pago un porcentaje de los créditos recibidos, hasta amortizar la deuda total.

En el caso de $E l$ Sol, los productores señalan que las evaluaciones de calidad no son muy justas, ya que en el proceso de selección, completamente manual, les rechazan producto que consideran reúne la calidad requerida. Sin embargo, permanecen con esta empresa porque a cambio de ello pueden tener los préstamos en efectivo ya señalados.

De acuerdo a la norma internacional, existen tres calidades para el pepinillo (primera, segunda y tercera) en función del diámetro que tengan, siendo los más angostos (hasta $1^{1 / 16}$ pulgadas de diámetro), los de primera calidad y mayor precio. Sin embargo, las empresas subdividen a cada una de esas calidades en otras dos y pueden demandar solo algunas de ellas. Para cumplir con esta demanda, los productores tienen que cosechar sus parcelas con cierta frecuencia, en ocasiones hasta diariamente, pues de un día para otro aumenta el tamaño del pepinillo y con ello el riesgo de que sea rechazado por la empresa. Pero aún cumpliendo con esa labor, los productores quedan con cierto porcentaje de producto grande, ya que el clima y otros elementos también influyen en la dimensión del producto final, el cual tienen que vender en el mercado nacional a precios insignificantes.

Los riesgos inherentes a la producción (clima y plagas) son asumidos completamente por los productores, lo que se agrava por el hecho de que éstos no aseguran el cultivo del pepinillo. En caso de siniestros, quedan endeudados con las empresas, por lo que tienen irles pagando en los siguientes ciclos productivos mediante entregas de producto.

Las ganancias de los productores por cultivar pepinillo se han venido reduciendo en los últimos años, debido al mayor incremento de los costos de producción, en relación al de los precios de venta, y al estancamiento de los rendimientos por 
hectárea. Aunque dichos costos dependen tanto del estado del cultivo (si hay plagas el gasto de insecticidas es mayor), como del rendimiento obtenido, en 2006 promediaron $\$ 37,640$ (2,308 euros) por hectárea. Aquellos ejidatarios que cultivan más de 5 hectáreas de pepinillo, lo cual rebasa su dotación legal de tierra, tienen que alquilar este recurso, por lo que a ese costo habría que añadir otros $\$ 3,500-\$ 4,000$ (214-245 euros) por la renta de una hectárea con agua, por el ciclo productivo de tres meses.

Sin embargo, el gasto más importante es el de la mano de obra, ya que se ocupa un promedio de 320 jornales por hectárea, cuyo gasto representa el $60 \%$ del costo total de producción (Cuadro 2). La labor más intensiva es la cosecha, durante la cual cada productor emplea al menos 15 peones diarios por hectárea, haciéndose un promedio de 20 cortes durante mes y medio que dura la cosecha. Esta es una labor muy meticulosa, en que solo deben seleccionarse los pepinos uniformes y de cierto tamaño y grado de maduración. Generalmente, el productor y/o algún familiar supervisan esta labor, además de que otros familiares trabajan también como cortadores. En la temporada de 2006 el jornal diario fue de \$100 (6.13 euros), el doble del pagado en 2001, y su tendencia es al ascenso, dado que cada vez la disponibilidad de mano de obra joven es menor, debido a la masiva migración a EU.

Cuadro 2. Valle de Santiago: costo de producción del pepinillo (2005).

\begin{tabular}{l|c}
\hline Labores & Pesos por hectárea \\
\hline Preparación del terreno (deshierbe, barbecho, diesel, tractor, chófer, etc.) & 1,500 \\
Semilla & 2,100 \\
Fertilizantes (urea, sulfato de amonio y fórmula 18-46) & 3,865 \\
Insecticidas (foliares) & 2,600 \\
Arpillas & 150 \\
Luz & 1,050 \\
Abejas & 575 \\
Mano de obra & 20,000 \\
Transporte parcela-centro de acopio & 1,800 \\
\hline Total & $\mathbf{3 3 , 6 4 0}$ \\
\hline
\end{tabular}

Fuente: trabajo de campo.

Otros rubros que han incrementado sus precios son los insumos químicos (fertilizantes e insecticidas), que entre 2001 y 2006 aumentaron 40\%, mientras que la tarifa eléctrica por la extracción de agua de pozo se incrementó en 2.5 veces en ese mismo lapso de tiempo (CFE, 2006). Comparativamente, los precios a los que compran el pepinillo las empresas no han aumentado en esa proporción. Durante 2004 y 2005, por ejemplo, el precio de la calidad más demandada permaneció sin variación (\$7.20 por kilo) (0.44 euros), y en 2006 aumentó a sólo \$7. 50 (0.46 euros). 
Además, en este año los productores dejaron de recibir bonificaciones o incentivos por calidad, debido, de acuerdo al gerente de una empresa, a la desfavorable situación del mercado (entrevista directa, 2006).

El promedio de rendimientos entre los pequeños productores es de 10 toneladas de pepinillo por hectárea, de las cuales el 60-70\% es de primera calidad. Aunque las ganancias por unidad de superficie varían de acuerdo a los rendimientos y calidad del producto, en promedio son del orden de \$10,000 (613 euros) por hectárea al año. Las 3 hectáreas que cultivan la mayoría de los ejidatarios les proporcionan por tanto un ingreso de $\$ 2,500$ (153 euros) mensuales, que aunque muy superior al salario mínimo legal, resulta insuficiente para el mantenimiento de una familia, que regularmente se integra por 6-8 miembros.

No obstante todo lo anterior, para los productores entrevistados existen ventajas de cultivar pepinillo bajo contrato. Una de ellas es el contar con un mercado más seguro, en comparación con sus posibles alternativas (hortalizas para el mercado interno o granos), en donde hay una mayor incertidumbre en los precios y toda una práctica de fraudes por parte de los compradores. Los productores señalan que aunque su relación con las empresas no es miel con hojuelas, y los precios del pepinillo no se han incrementado como debieran, al menos tienen una mayor seguridad en lo que a pagos se refiere. Otra ventaja es el acceso a créditos para poder emprender cultivos que son caros, como es el caso de las hortalizas en general y del pepinillo en particular. Como vimos, la mano de obra, lo más costoso de este cultivo, la van pagando los productores con las liquidaciones semanales que las empresas les hacen por su producto entregado.

El financiamiento que otorgan las empresas es crucial, dado que no existe este apoyo por parte del gobierno para pequeños productores de hortalizas, y la banca privada solo lo concede a grandes productores, quienes son los que pueden cumplir con las garantías requeridas. Las cajas de ahorro popular, que están siendo la alternativa de financiamiento para muchos productores, no otorgan créditos que cubran la totalidad del costo del cultivo.

Por otra parte, los productores obtienen con el pepinillo cerca del doble de ganancias por unidad de superficie en comparación con los granos, además de que éstos tienen un ciclo productivo más largo (seis meses), durante el cual pueden establecerse dos ciclos de hortalizas. De hecho, la gran mayoría de los productores de pepinillo, al no poder subsistir exclusivamente de este cultivo, siembran también brócoli fresco de exportación, el cual producen bajo contrato para las empresas ubicadas en el municipio (ver Echánove, 2006). Cultivan el pepinillo durante el período de veda o prohibición oficial del brócoli (febrero-mayo), estableciendo un total de tres ciclos de estas hortalizas al año: pepinillo-brócoli-brócoli. Algunos productores, generalmente los que cultivan entre 5 y 10 hectáreas, también destinan pequeñas superficies a otras hortalizas (chícharo, calabaza, coliflor, etc.).

Paralelamente, la mayoría de los pequeños productores cultivan granos para su autoconsumo (maíz y frijol) o para la alimentación de sus animales (engorda de 
cerdos), motivados también por conservar el apoyo gubernamental del Procampo y realizar la necesaria rotación de suelos requerida por el cultivo de hortalizas.

Aún así, el grueso de los ejidatarios solo ha logrado como producto de su actividad agrícola modestas mejoras en su nivel de vida (vivienda, alimentación, etc.), logrando comprar, en el mejor de los casos, maquinaria y animales de trabajo. Sin embargo, existen algunos casos de productores que han transitado a pequeños empresarios, basados en una mayor diversificación productiva y escala de operación, ya que mediante la renta y/o compra de tierras y/o mediería cultivan superficies de al menos 50 hectáreas.

Consecuentemente, para poder subsistir, los pequeños productores de hortalizas han tenido que diversificar sus ingresos, gran parte de los cuales ya no se origina en la actividad agropecuaria, al igual que lo ocurrido en el resto del país. Todo el que ha podido ha abierto una tienda de víveres en su pueblo y los jóvenes que no han migrado se emplean en maquiladoras de ropa o agroindustrias de la región. Sin embargo, la principal fuente de ingreso extra-agrícola es el envío de remesas de familiares que migraron a EU, de tal manera que hoy día es difícil encontrar una familia en Valle en la que no exista al menos un miembro trabajando del otro lado de la frontera norte. Este hecho no es privativo de este municipio sino que es generalizable al medio rural mexicano, de tal modo que hoy día las remesas constituyen la segunda fuente de divisas del país, antecedida tan solo por las exportaciones de petróleo.

\section{Reflexiones finales}

La agricultura por contrato ofrece importantes ventajas para las empresas, entre las que figuran control sobre la oferta (cantidad y calidad), menores costos y riesgos, mayor expansión y diversificación de sus operaciones, obtención de apoyos gubernamentales, mayor movilidad geográfica y acceso a mano de obra flexible y calificada (Raynolds, 1997; Singh, 2002; Brannstrom, 2000: 329, 337; Dolan, 2001: 44; Cook, 1994: 234; Echánove, 2005, 2006).

Los resultados o efectos de los contratos en los productores dependen de las relaciones de poder entre éstos y las empresas y varían de acuerdo a las estructuras agrarias y de mercado y al entorno socio-económico y político del país (Porter y Phillips-Howard, 1997: 229; Masakure y Henson, 2005: 1732). Otros elementos determinantes son el acceso de los productores a recursos como mano de obra, agua, transporte y tierra; las características de sus familias (Masakure y Henson, 2005: 1729); la competencia existente entre las empresas; la reglamentación oficial sobre las relaciones entre éstas y los productores; la participación de organismos privados o ONG's; la organización y fuerza de los productores, y la escala de la operación de éstos. En México no existen leyes ni instancias que regulen el cumplimiento de los contratos, como tampoco organización por parte de los productores de pepinillo. 
Los agricultores buscan a través de los contratos mejorar su ingreso, tener un mercado seguro, disminuir los riesgos de fluctuaciones de precios y acceder a capital, tecnología e insumos. Masakure y Henson (2005: 1726) señalan que esos agentes también se contratan porque no tienen mejores alternativas de ingreso, como sucede en el caso del pepinillo aquí presentado. En este, se evidenciaron las desventajas que los contratos han tenido para los productores y que son similares a las reportadas en muchos otros estudios de caso (Clapp, 1994; Kay, 1997; Singh, 2002, 2005; Murray, 2002; Glover y Kusterer, 1990; Echánove, 2001, 2005, 2006). Singh (2002:1624), por ejemplo, señala que las empresas otorgan un deficiente servicio de extensión, cargan precios excesivos por sus servicios, no compensan a los productores por riesgos climáticos, ofrecen bajos precios por el producto, favorecen a los productores grandes, dilatan los pagos y no explican a los productores los mecanismos de fijación del precio. A esto habría que añadir el aspecto de la calidad, cuyos requerimientos, como señala Watts (1994: 65), son cambiantes y constituyen un mecanismo de regulación de la oferta por parte de las empresas. Otras desventajas de los contratos es que éstos están diseñados para proteger a las empresas, ya que, como señala Singh (2002: 1632), son obligatorios para los productores, pero no especifican ninguna responsabilidad de la compañía ante la falla de comprar el producto.

A pesar de todo ello, diversos estudios de caso han demostrado mejoría en el ingreso de los productores contratados y en el empleo (Little, 1994; Collins, 1993: 77; Brannstrom, 2000: 337; Singh, 2002: 1632; Key y Runsten, 1999; Gwynne, 1999: 221223; McCulloch y Masako, 2002). Lo mismo ha sucedido entre los productores de pepinillo estudiados, quienes estarían en peores condiciones si no se hubiesen contratado para las empresas. Sin embargo, como hemos visto, la mayoría de ellos sigue siendo pobre, aunque evidentemente su reducida escala de operación (3-5 hectáreas) hace difícil su tránsito a otro estadio económico.

El tipo de productores con que contratan las empresas depende de diversos factores, como las características del cultivo y sus demandas laborales, tamaño de productores existentes en la región de influencia de las agroindustrias, costos de transacción de éstas, etc. Las empresas escogen cuidadosamente a sus productores, privilegiando a los capitalizados, que son los que ofrecen mejor calidad, cuentan con infraestructura (bodegas, maquinaria, transporte) y, sobre todo, les representan menores costos de transacción. Dentro de éstos, son importantes para las empresas los relacionados con el traslado de sus técnicos o supervisores, el cual es menor cuando atienden a pocos productores con superficies compactas que si tienen que acudir con un gran número de agricultores chicos y dispersos geográficamente. Sin embargo, también existe contratación con pequeños productores, como lo han demostrado tanto el caso del pepinillo, como de otros en diversos países de Asia, América Latina y el Caribe (Grossman, 1998; Swinnen y Maertens, 2006; Collins, 1993; Glover y Kusterer, 1990; Korovin, 1992; Murray, 2001; Raynolds, 1997; Little y Watts, 1994; Vellema, 2002; Gwynne y Kay, 1997; Masakure y Henson, 2005; Burch, 1996). 
En ocasiones se explica porque las empresas no tienen otra alternativa más que contratar a pequeños productores, ya que son los que se encuentran cercanos a la empresa, pero en la mayoría de los casos la razón es que se trata de cultivos familiares, muy intensivos en mano de obra. En el caso del pepinillo, los dos factores señalados explican la presencia de pequeños productores.

No puede negarse que el cultivo de hortalizas ha generado empleos en Valle, sin embargo, no todos los pequeños productores pueden cultivarlas, dado que, o sus parcelas no cuentan con agua, o no tienen los recursos necesarios para rentar tierras que cuenten con ella. Por otra parte, las superficies cultivadas con pepinillo se han venido reduciendo en los últimos ciclos productivos, debido a la menor participación de México en las importaciones estadounidenses, como efecto de la competencia con India. Además, EU sigue siendo un importante productor de pepinillo y sus compras externas solo representan alrededor del 6\% de su consumo, el cual se ha estancado durante los últimos años, por lo que se considera como un mercado "maduro".

La creciente participación de India, que exporta a granel pepinillos en salmuera, ha dado lugar al desplome de los precios internacionales, cuyos niveles actuales son el 60\% de los que prevalecían hace diez años (Veerakumar, 2006). La mayor competencia y la saturación de los mercados de destino es a lo que comúnmente se enfrentan los productos llamados no-tradicionales, los que, después de un período de auge, entran en fases de estancamiento (Echánove, 2005). Ante ello, algunos autores han planteado que a largo plazo la sustentabilidad económica de esos bienes está basada en producir calidad y añadir valor. Sin embargo, la cuestión resulta más compleja y lleva al cuestionamiento de los modelos de desarrollo orientados a la exportación, implementados por los países del Tercer Mundo a partir de los ochenta, y que en muchos países han probado ser muy vulnerables. Sin dejar de trabajar por el incremento de la calidad y el valor agregado de los bienes exportados, es también prioritario el fortalecimiento de los mercados internos, lo que requiere de políticas de desarrollo diferentes a las planteadas por los modelos de corte neoliberal.

Finalmente, hay que señalar que el cultivo de hortalizas en Valle ha dibujado ya sus límites. La proliferación incontrolada de plagas ha sido tan extrema, que desde hace años existen períodos de veda o prohibición del cultivo de la principal hortaliza, el brócoli. Tanto en ésta como en el pepinillo, el uso de pesticidas peligrosos afecta cotidianamente la salud humana y contribuye a la elevada contaminación de suelos y aguas existentes. Asimismo, ha habido una excesiva extracción de agua del subsuelo para riego, con el consecuente abatimiento de los acuíferos (a una tasa de 3 metros por año) y salinización de los suelos (Alcocer et al., 2000). Todos estos efectos en el medio ambiente son un claro cuestionamiento a la sustentabilidad de la actividad aquí estudiada. 


\section{Bibliografía}

Alcocer, J., Escobar, E., y Lugo, A. (2000) Water use (and abuse) and its effects on the crater-lakes of Valle de Santiago, Mexico. Lakes and Reservoirs: Research and Management 5, 145-149.

Brannstrom, C. (2000) Coffee labour regimes and deforestation on a Brazilian frontier, 1915-1965. Economic Geography 76, 326346.

Burch, D. (1996) Globalized agriculture and agri-food restructuring in southeast Asia The Thai experience. En Burch, D., Rickson, R. E., y Geoffrey, L. (eds) Globalization and Agri-Food Restructuring. Perspectives from the Australasia Region. England: Avebury, p. 323-344.

CFE (Comisión Federal de Electricidad) (2006) Estadisticas de precios de la tarifa 9M. $<$ http://aplicaciones.cfe.gob.mx/>.

Clapp, R. (1994) The Moral Economy of the Contract. En Little, P.D. y Watts, M. (eds) Living under contract. Contract farming and agrarian transformation in SubSabaran Africa. Madison: The University of Wisconsin Press, p. 78-94.

Collins, J. (1993) Gender, Contracts and Wage Work: Agricultural Restructuring in Brazil's Sao Francisco Valle. Development and Change 24, 53-82.

Cook, I. (1994) New Fruits and Vanity: Symbolic Production in the Global Food Economy. En Bonnano et.al. (eds) From Columbus to Conagra. The Globalization of Agriculture and Food. Lawrence, Kansas: University Press of Kansas, p. 232248.

Dolan, C. (2001) The 'Good Wife': Struggles over Resources in the Kenyan Horticultural Sector. The Journal of Development Studies 37 (3), 39-70.

Echánove, F. (2001) Working Under Contract for the Vegetable Agroindustry in Mexico: A Means of Survival. Culture and Agriculture 23 (3), 13-23.
Echánove, F. (2005) Globalisation and Restructuring in Rural Mexico: the Case of Fruit Growers. Tijdschrift voor Economische en Sociale Geografie 96 (1), 15-30.

Echánove, F. (2006) Contract Farming and Small-Scale Producers: Nontraditional Vegetable Exports from Mexico. Nordic Journal of Latin American and Caribbean Studies XXXVI: 1, 83-102.

Echánove, F. y Steffen, C. (2005) Agribusiness and Farmers in México: the Importance of Contractual Relations. The Geographical Journal 171 (2), 166-176

Glover D. y Kusterer K. (1990) Small Farmers, Big Business. Contract Farming and Rural Development. New York: St. Martin Press.

Grossman, L. (1998) The Political Ecology of Bananas. Contract Farming, Peasants, and Agrarian Change in the Eastern Caribbean. Chapel Hill: University of North Carolina Press.

Gwynne, R. (1999) Globalisation, commodity chains and fruit exporting regions in Chile. Tijdschrift en Economische en Sociale Geografie 90, 211-225

Gwynne, R. y Kay, C. (1997) Agrarian Change and the democratic transition in Chile. Bulletin of Latin American Research 16 (1), 3-10

Kay, C. (1997) Globalisation, Peasant Agriculture and Reconversion. Bulletin of Latin American Research 16, 11-24.

Key, N. y Runsten, D. (1999) Contract farming, smallholders and rural development in Latin America: the organization of agroprocessing firms and the scale of outgrower production. World Development 27 (2), 381-401.

Korovin, T. (19929 Peasants, Grapes and Corporations. The Growth of Contract Farming in a Chilean Community. The Journal of Peasant Studies 19 (2), 228-54.

Little, P. (1994) Contract Farming and the Development Question. En Little, P. y 
Watts, M. (eds) Living under Contract. Contract Farming and Agrarian Transformation in Sub-Sabaran Africa Madison: The University of Wisconsin Press, p. 216-247.

Little, P. y Watts, M. (eds) (1994) Living under Contract. Contract Farming and Agrarian Transformation in Sub-Saharan Africa. Madison: The University of Wisconsin Press.

Masakure, O. y Henson, S. (2005) Why Do Small-Scale Producers Choose to Produce under Contract? Lessons from Nontraditional Vegetable Exports from Zimbabwe. World Development 33 (10), 17211733.

McCulloch, N. y Masako, O. (2002) Export horticulture and poverty in Kenya. Working Paper 174. Brighton UK: Institute of Development Studies.

Murray, W. (2001) Dilemmas of development in Oceania: the political economy of the Tongan agro-export sector. The Geographical Journal 167 (4), 291-304.

Murray, W. (2002) From Dependency to Reform and Back Again: The Chilean Peasantry During the Twentieth Century. The Journal of Peasant Studies 29 (3-4), 190-227.

Perry, J. y Banker, D. (2000) Contracting Changes How Farm Do Business. En Rural Conditions and Trends, vol. 10, no. 2. ERS-USDA.

Porter, G. y Phillips-Howard, K. (1997) Comparing Contracts: An Evaluation of Contract Farming Schemes in Africa. World Development 25 (2), 227-238.

Raynolds, L. (1997) Restructuring National Agriculture, Agro-Food Trade and Agrarian Livelihoods in the Caribbean. En Goodman y Watts (eds) Globalising Food. Agrarian Questions and Global Restructuring. London and New York: Routledge. p. 119-132.

Raynolds, L. (2002) Wages for Wives: Renegotiating Gender and Production Relations in Contract Farming in the
Dominican Republic. World Development 30 (5), 783-798.

Roy, P. (1972) Contract Farming and Economic Integration. Danville, IL: Interestate Press.

Saenz, F. y Ruben, R. (2004) Export contracts for non-traditional products: Chayote from Costa Rica. Journal on Chain and Network Science 4, 139-150.

SAGARPA (Secretaría de Agricultura, Ganadería, Desarrollo Rural, Pesca y Alimentación) (2007) CADER Valle de Santiago, Costos de producción del pepinillo.

SAGARPA (Secretaría de Agricultura, Ganadería, Desarrollo Rural, Pesca y Alimentación) (2008) Sistema de Información Agropecuaria de Consulta $<$ http://www.siap.gob.mx/>.

Schwentesius R. y Gómez, M. (1998) Competitividad de hortalizas mexicanas en el mercado norteamericano. Tendencias recientes en el marco del TLC. En Schwentesius R., Gómez, M. y Williams, G. (coord), TLC y Agricultura ¿Funciona el experimento? México: Juan Pablos. pp. 167-203.

Singh, S. (2002) Contracting Out Solutions: Political Economy of Contract Farming in the Indian Punjab. World Development 30 (9), 1621-1638.

Singh, S. (2005) Contract faming in Thailand: An Overview. <www.irma.ac.in/pub/network/sukh.html> Swinnen, J. F. y Maertens, M. (2006) Globalization, Privatization and Vertical Coordination in Food Value Chains in Developing and Transition Countries. Ponencia presentada en la $26^{\text {th }}$ Conferencia de la Asociación Internacional de Economistas Agrícolas, Queensland, Australia, Agosto 12-18.

USDA (United States Department of Agriculture) (2008)

$<$ http://www.fas.usda.gov/ustrdscripts/US Report.exe>. 
Veerakumar, M. (2006) Importation of Raw Products for the US Pickle Industry. Cucurbit Breeding $<$ http://cuke.hort.ncsu.edu/cucurbit/cuke/ ppi01abst/ppiveerakumar.html>

Vellema, S. (2002) Making contract farming work? Society and technology in Philippine transnational agribusiness. Maastricht: Shaker Publishing.
Watts, M. (1994) Life under Contract: Contract Farming, Agrarian Restructuring and Flexible Accumulation. En Little y Watts (eds.) Living under contract. Contract farming and agrarian transformation in Sub-Sabaran Africa. Madison, Wisconsin: The University of Wisconsin Press. p. 2177. 